\title{
An Energy-Based Low-Order Approach for Mission Analysis of Air Vehicles in LEAPS
}

\author{
Francisco M. Capristan* and Jason R. Welstead* \\ NASA Langley Research Center, Hampton, Virginia, 23681, USA
}

\begin{abstract}
Mission analysis methods that allow rapid analysis of conceptual aircraft provide great benefits to designers because a large number of feasibility and optimization studies can be performed quickly. This paper presents a novel low-order mission analysis approach that will be included in a new aircraft analysis tool called the Layered and Extensible Aircraft Performance System, also known as LEAPS. This approach is based on the mission analysis method used in the Flight Optimization System, also known as FLOPS. FLOPS has been developed at NASA Langley Research Center for over 30 years, and it is mainly used to analyze conventional aircraft (e.g., gas turbine-powered aircraft). One of the main features of FLOPS is its ability to perform rapid mission analysis. This quick analysis turnaround makes FLOPS a suitable tool to perform a large number of trade studies and optimizations. For this reason, the main concepts applied in FLOPS will be used and expanded in LEAPS to allow the analysis of unconventional aircraft that use novel propulsion systems. This expanded approach includes the capability to handle electric propulsion systems as well as multiple propulsor classes at the same time. A novel hybrid-electric aircraft configuration is used to investigate the potential benefits of the proposed mission analysis approach. The results show that the design space increases which can potentially lead to better designs.
\end{abstract}

\section{Nomenclature}

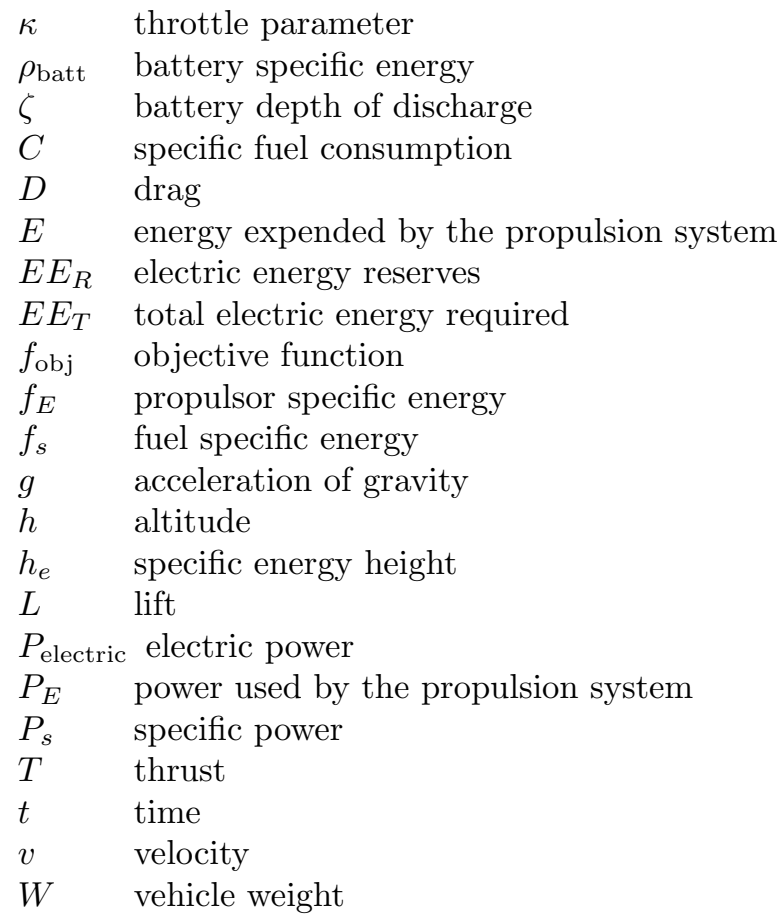

*Aerospace Engineer, Aeronautics Systems Analysis Branch, 1 N Dryden St. M/S 442, Hampton VA 23681, AIAA Member. 
$W_{\text {batt }}$ battery weight

$W_{f} \quad$ fuel weight

\section{Introduction}

The Layered and Extensible Aircraft Performance System (LEAPS) is a new aircraft analysis tool currently being developed by members of the Aeronautics Systems Analysis Branch (ASAB) and the Vehicle Analysis Branch (VAB) at NASA Langley Research Center. ${ }^{1}$ Historically, ASAB has used the Flight Optimization System (FLOPS) ${ }^{2,3}$ as its main aircraft analysis tool. FLOPS is well suited to study conventional aircraft but lacks capabilities to handle the novel configurations and propulsion systems currently being proposed by NASA, industry, and academia. This is the main reason behind the development of LEAPS. LEAPS is being written in Python 3.x and it will handle novel configurations by providing the flexibility required to add custom-built analysis modules. LEAPS will also have a multi-order analysis approach that will provide a balance between computational cost and analysis fidelity level. ${ }^{1}$

FLOPS was initially developed over 30 years ago, and it was constrained by the computational capabilities available at that time. For that reason, the algorithms in FLOPS are designed to use a limited amount of memory and computational power. In general, the methods used in FLOPS are considered to be low-order when compared to state-of-the-art methods that often require significant computing resources. Despite their low-order label, these algorithms have been successfully used for over 30 years because they provide reasonable results in a timely manner with limited computer power. The overall increase in computation power experienced in the last 30 years has made FLOPS a suitable tool to perform a large number of trade studies and optimizations because a single analysis often takes less than a couple of seconds. In contrast, some newer aircraft analysis tools designed to study unconventional vehicles can take minutes to return a single analysis. For this reason, the LEAPS team decided to include the appropriate FLOPS methodologies in the new analysis tool. Furthermore, these methodologies are being enhanced to ensure that LEAPS will be able to analyze unconventional propulsion systems (e.g., hybrid-electric aircraft).

The multi-order nature of LEAPS will allow the user to select the order (low and higher-order) of the mission analysis approach. ${ }^{4}$ In particular, this paper presents the low-order mission analysis approach currently being considered for LEAPS. This mission analysis procedure is based on the methodology used in FLOPS and will allow LEAPS to have FLOPS-like capabilities when a low-order mission analysis approach is desired. It is important to note that the LEAPS mission analysis approach is not a simple rewrite of FLOPS; it is a complete overhaul that includes the appropriate capabilities to analyze unconventional concepts.

\section{Background}

\section{A. Energy Method}

FLOPS uses the energy method to analyze the climb and descent segments in a mission. ${ }^{2}$ The energy method was first introduced by Rutowski. ${ }^{5}$ Raymer $^{6}$ discussed some of its practical aspects and provided some examples. A previous study performed by the authors concluded that the energy method provides useful trajectories without the need to solve the equations of motion for the vehicle. ${ }^{4}$ This method is based on the exchange between kinetic and potential energy. To highlight its main concepts, consider the sum of the kinetic and potential energy normalized by the vehicle weight, known as the specific energy height $\left(h_{e}\right)$

$$
h_{e}=h+\frac{1}{2 g} v^{2}
$$

where $h$ is the altitude, $v$ is the velocity magnitude, and $g$ is the acceleration due to gravity. The excess specific power available to change energy heights is given by

$$
P_{s}=\frac{v}{W}(T-D)=\frac{\mathrm{d} h_{e}}{\mathrm{~d} t}
$$

where the vehicle thrust is specified by $T$, the drag by $D$, and vehicle weight by $W$. Consequently, the time required to change from one specific energy height to another is given by

$$
t_{1-2}=\int_{h_{e_{1}}}^{h_{e_{2}}} \frac{1}{P_{s}} \mathrm{~d} h_{e}
$$


Maximizing $P_{s}$ will minimize the time required to change the specific energy heights. A similar approach is used to minimize the fuel required to change energy heights. Consider the fuel specific energy as

$$
f_{s}=\frac{P_{s}}{C T}=\frac{\mathrm{d} h_{e} / \mathrm{d} t}{\mathrm{~d} W_{f} / \mathrm{d} t}
$$

where $C$ is the specific fuel consumption. The change in fuel weight is given by

$$
W_{f_{1-2}}=\int_{h_{e_{1}}}^{h_{e_{2}}} \frac{1}{f_{s}} \mathrm{~d} h_{e}
$$

Similarly, the fuel weight needed to change states is minimized when $f_{s}$ is maximized.

Reference (4) shows an expression that allows the energy method to focus on energy used by the propulsion system instead of fuel. This provides a suitable approach to provide trajectories that minimize energy usage; thus, it is suitable for electric and hybrid-electric propulsion aircraft. This extension uses the rate of change in energy levels relative to the power required to drive the engines. This ratio is defined as the propulsor specific energy, $f_{E}$, and is given by

$$
f_{E}=\frac{P_{s}}{P_{E}}=\frac{\mathrm{d} h_{e} / \mathrm{d} t}{\mathrm{~d} E / \mathrm{d} t}
$$

where $P_{E}$ is the power used by the propulsion system, and $E$ is the energy expended by the propulsion system. The required energy input to the propulsion system to change energy heights is given by

$$
E_{1-2}=\int_{h_{e_{1}}}^{h_{e_{2}}} \frac{1}{f_{E}} \mathrm{~d} h_{e}
$$

The energy required by the propulsion system is minimized when $f_{E}$ is maximized.

\section{B. Mission Analysis Approach in FLOPS}

In order to present a low-order mission analysis approach appropriate for LEAPS, it is necessary to first understand the approach used in FLOPS and identify areas that need improvement.

\section{Assumptions}

FLOPS uses the energy method to determine the appropriate climb and descent trajectories. It uses steady, level flight assumptions for cruise segments. There are numerous simplifying assumptions and other considerations used in FLOPS, some of these include:

- vehicle modeled as a point mass (no rotational degrees of freedom),

- methodology designed for consumable fuel aircraft but possible to analyze hybrid-electric vehicles by performing a series of workarounds,

- all engines are the same (one engine data set per analysis),

- different engines cannot be throttled independently, and

- angle of attack not considered after takeoff.

These assumptions allow for quick analysis of missions; however, they also restrict the types of vehicles and/or missions that can be analyzed. For example, FLOPS cannot analyze vertical take-off and/or landing segments or throttle propulsors independently. Some workarounds have been used in the past to analyze electric and hybrid-electric vehicles with FLOPS, but they often result in complex, one-off architectures that are designed for a specific vehicle. ${ }^{7,8}$ 


\section{Mission Integration}

FLOPS determines the trajectories to be flown by creating a series of lookup tables for the different segments (e.g., climb, cruise, descent). These tables are generated by maximizing certain parameters in the energy method (e.g., specific excess power) or other cruise conditions. The lookup tables are then treated as surrogate models that predict the optimum flight conditions. The algorithms in FLOPS then iterate through the different surrogate models to find the optimal trajectory path.

For a climb segment, the analyst can specify a combination of minimum time-to-climb or minimum fuelto-climb profiles. FLOPS generates a grid of specific energy heights (Eq. 1) and vehicle weights. Flight conditions at each grid point are adjusted to ensure that the parameter of interest is optimized. A similar approach is taken for the descent segment, where a common variable to maximize is the lift-to-drag ratio, $L / D$.

For a cruise segment, the analyst has different options to obtain the desired trajectory. These options include:

- optimum altitude and Mach number for specific range,

- fixed Mach number, optimum altitude for specific range,

- fixed altitude, optimum Mach number for specific range,

- fixed altitude, optimum Mach number for endurance,

- fixed altitude, constant lift coefficient,

- fixed Mach number, optimum altitude for endurance,

- optimum Mach number and altitude for endurance,

- maximum Mach number at input fixed altitude,

- maximum Mach number at optimum altitude, and

- fixed Mach number, constant lift coefficient.

Multiple cruise profiles can be specified during an evaluation. For each potential profile, FLOPS generates an array of expected vehicle weights. One optimization takes place for each vehicle weight in the array and the results are stored to be accessed as a lookup table. This lookup table is then used and interpolated to provide the optimal or desired conditions for a given vehicle weight.

\section{Low-Order Mission Analysis Approach (L0 Approach)}

The mission analysis approach presented in this paper is inspired by the method used in FLOPS and is referred as the L0 mission analysis approach. FLOPS uses internal interpolation and optimization methods, while the L0 mission analysis approach relies on SciPy's algorithm. ${ }^{9}$ In particular, the Sequential LeastSquares Quadratic Programming ${ }^{10}$ (SLSQP) algorithm is used to perform all the optimizations. The main additions/modifications to the FLOPS method are:

- method can handle electric and hybrid-electric engines,

- multiple engine data sets can be used at the same time,

- propulsors can be throttled independently of each other,

- objective function for optimal trajectory is given by the user,

- battery weight estimate (if applicable) is included during the mission iteration, and

- flexible architecture that allows radical vehicle changes in-between segments.

Note that the term battery is used in this paper to refer to any type of constant weight electrical energy storage device. The following subsections present the main ideas used in the development of the L0 mission analysis approach. 


\section{A. Climb and Descent Analysis}

The energy method (discussed in Section II-A) is used to determine the climb and descent trajectories. Every climb and descent trajectory has specific energy height bounds based on the initial and final desired state vectors. An estimate of the lower and upper bounds for the vehicle weight can be provided by a weights module or given by the analyst. The estimate should include an initial conservative guess for the battery weight. The possible vehicle weights and specific energy levels are discretized to form a grid. Each point in the grid is optimized given the desired objective function. The optimization problem for a given weight, $W_{j}$, and specific energy height, $h_{e_{i}}$, is expressed as:

$$
\begin{array}{cl}
\underset{h, \vec{\kappa}}{\operatorname{minimize}} & f_{\mathrm{obj}} \\
\text { subject to } & h+\frac{v^{2}}{2 g}=h_{e_{i}} \\
& h_{\min } \leq h \leq h_{\max } \\
& 0 \leq \vec{\kappa} \leq 1
\end{array}
$$

where $f_{\text {obj }}$ is the objective function that can represent the minimum time-to-climb (Eq. 2), minimum fuelto-climb (Eq. 4), minimum energy-to-climb (Eq. 6), or any combination of them. In this formulation, the optimizer controls the altitude, $h$, and the vector of throttle parameters for each propulsor class. $\kappa$. Similarly, a common approach for the descent segment involves having an unpowered segment where the objective is to maximize $L / D$. Note that speed limits at certain altitudes due to regulations can be easily incorporated by adjusting the altitude at a given specific energy height (no optimization necessary). The optimization problem is repeated for all $i, j$ points in the grid. This grid is used as a surrogate model that contains the optimal conditions for the segment.

\section{B. Cruise Analysis}

Steady flight conditions are assumed for the cruise segment. It is assumed that thrust equals drag and that lift equals weight. For fixed conditions (constant altitude and velocity), it would be possible to obtain accurate results by simply discretizing the Breguet-range equation; however, it is possible that optimal flight conditions might change as the vehicle changes weight. For this reason, the weight is discretized in a similar manner as in Section III-A, and an optimization process is used for each weight, $W_{j}$. The optimization formulation takes the following form:

$$
\begin{array}{cl}
\underset{h, v, \vec{\kappa}}{\operatorname{minimize}} & f_{\mathrm{obj}} \\
\text { subject to } & T-D=0 \\
& L-W_{j}=0 \\
& h_{\min } \leq h \leq h_{\max } \\
& v_{\min } \leq v \leq v_{\max } \\
& 0 \leq \vec{\kappa} \leq 1
\end{array}
$$

where $f_{\text {obj }}$ is specified by the user. For conventional propulsion systems (i.e., consumable fuel), the desired objective functions are well understood. Maximum endurance can be obtained by minimizing the fuel flow rate at the given instant and maximum range can be obtained by minimizing the ratio of fuel-flow rate to the velocity. These objective functions cannot be used if the vehicle uses propulsion systems that do not rely on consumable fuel. For this reason, the fuel-flow rate is replaced by the power required to drive the propulsion system. By using this approach, it is possible to get maximum range and endurance missions for electric or hybrid-electric aircraft. Table 1 shows a set of possible objective functions for the cruise segment. Analyst can include their desired objective function; thus, other parameters of interest could be used when desired. 
Table 1. Possible Objective Functions for the Cruise Segment

\begin{tabular}{ccc} 
& fuel-based & power-based \\
\hline \hline Maximum Range & $\frac{1}{v}\left(\frac{\mathrm{d} W_{f}}{\mathrm{~d} t}\right)$ & $\frac{P_{E}}{v}$ \\
Maximum Endurance & $\frac{\mathrm{d} W_{f}}{\mathrm{~d} t}$ & $P_{E}$
\end{tabular}

\section{Mission Analysis Integration}

The climb, descent, and cruise optimization tables or surrogate models (discussed in the sections above) are used to determine the trajectory profile. Figure 1 shows a high-level overview of how the trajectory is adjusted until it converges. The following steps present the logic used to determine a trajectory that includes a climb, cruise, and descent segment for a vehicle that uses both fuel-based and electric propulsion systems.

1. Specify desired range, flight conditions for each segment, empty weight, and a conservative battery weight guess,

2. Start from the descent segment and integrate backwards in time until conditions at cruise are intersected by looking at the altitude and weight in the cruise segment surrogate.

3. Integrate cruise until desired cruise range is obtained and provide final conditions for climb segment.

4. Integrate climb until takeoff conditions are reached.

5. Compute battery weight required and total range.

6. Compute differences between calculated range and battery weight with the previous guess

7. Adjust cruise range and battery weight appropriately.

8. Repeat steps 2 to 7 until the desired total range is achieved and battery weight converges.

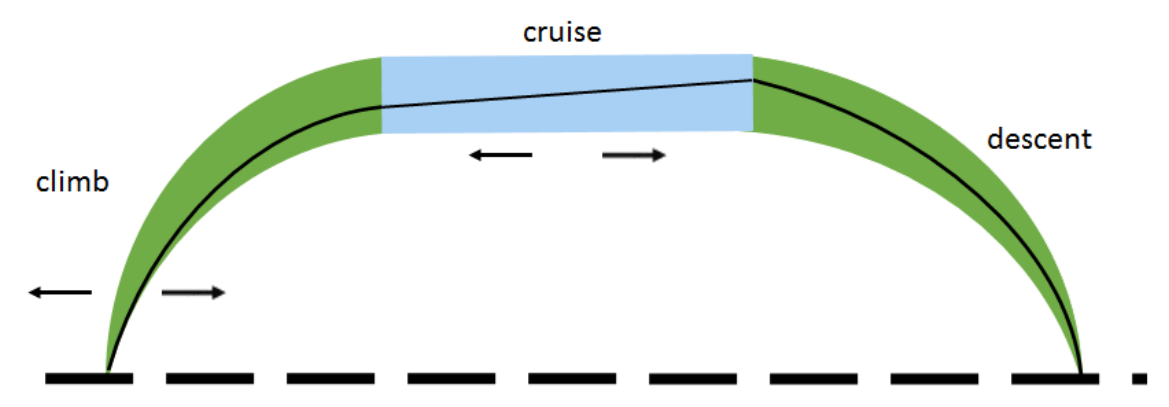

Figure 1. High-level representation of integration of the LO mission analysis approach. The colored segments represent the different trajectory possibilities due to the possible vehicle weights. The cruise segment can be elongated or shortened while the climb segment is shifted to the left or right to meet the desired range. The solid black line represents the optimal trajectory given a final weight. The process is iterated until the battery weight converges and the desired range is achieved. The optimal trajectory can change in every convergence iteration due to weight changes.

Reference (4) shows in more detail how to obtain a full trajectory that includes velocities, altitudes, and distance traveled for the segments that use the energy method. The energy method in between segments (e.g., cruise and descent) is used to obtain the time required for the vehicle to connect the optimal velocities and/or altitudes in each segment.

The battery is sized by keeping track of the electric power at each instance. The total electric energy required, $E E_{T}$, is computed by

$$
E E_{T}=\int_{t_{0}}^{t_{f}} P_{\text {electric }} d t
$$

$$
6 \text { of } 12
$$


where the electric power is given by $P_{\text {electric }}$. The battery is sized by ensuring that it will store the necessary electric energy. The battery weight can be computed by

$$
W_{\text {batt }}=\frac{\left(E E_{T}+E E_{R}\right) / \zeta}{\rho_{\text {batt }}}
$$

where $E E_{R}$ is the reserve electric energy, $\zeta$ is the maximum allowable battery depth of discharge, and $\rho_{\text {batt }}$ is the specific energy of the battery.

\section{Mission Analysis of a Hybrid-Electric Aircraft}

The vehicle selected to test the L0 mission analysis capability is the Parallel Electric-Gas Architecture with Synergistic Utilization Scheme (PEGASUS) concept. This vehicle uses multiple electric and hybridelectric propulsors to obtain aerodynamic benefits. Antcliff and Capristan ${ }^{8}$ showed that this vehicle has the potential to be more energy efficient than similar vehicles that use only gas engines.

PEGASUS uses three different propulsor classes as shown in Fig. 2. Parallel hybrid-electric propulsors are located at the wingtips, two electric propulsors are located inboard on the wing to provide additional propulsion during takeoff and climb, and a final electric propulsor is located at the tail. Reference (8) provides a detailed description of the potential benefits of this configuration and the analysis challenges encountered. FLOPS was used to analyze this configuration, and due to its limitations multiple workarounds were required. The limitations include the lack of the proper framework to analyze three different propulsors at the same time. Also, the inboard propellers can be folded midflight; the folded configuration affects the aerodynamics of the vehicle and FLOPS cannot fully handle drag changes due to the thrust generated. The complexities in the PEGASUS vehicle make it a suitable test case to investigate the features being considered in LEAPS. This section presents the differences in analyzing this configuration with FLOPS and the L0 mission analysis approach. The mission investigated has a required range of 400 nautical miles at 20,000 feet and 300 knots.

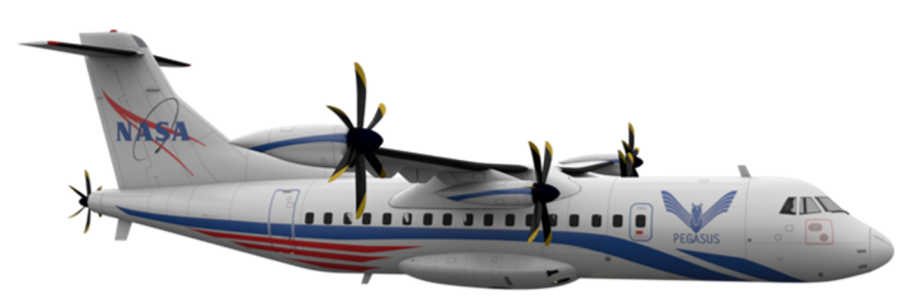

(a) Artist depiction

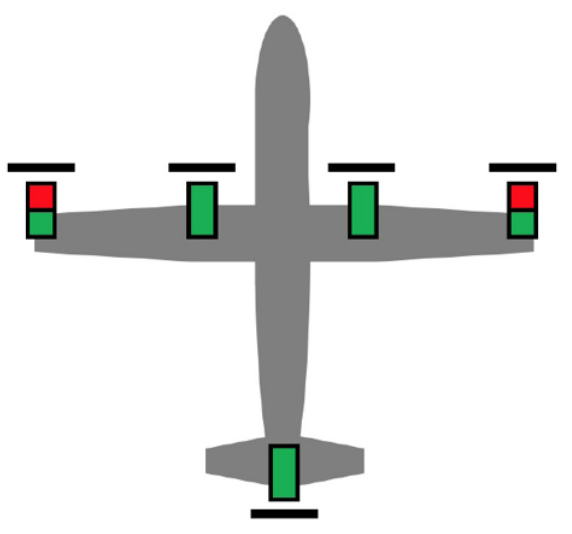

\section{日: Turbine-Electric Motor ए: Electric Motor}

(b) Propulsor configuration

Figure 2. PEGASUS concept

\section{A. Comparison with FLOPS}

The limitations in FLOPS force the analyst to aggregate all propulsors into a single engine data set. A data set provides thrust, fuel flow, and the electric energy used given the flight conditions and throttle parameter. One of the main limitations of this approach is the lack of freedom to throttle the propulsors independently. For example, FLOPS cannot identify (given an objective function) if a propulsor should be at 100\% throttle setting while the others are at idle or folded. In this test case, a single engine data set containing the information for all three propulsor types was used. The inboard propulsors were adjusted to behave as if 
they are folded during cruise (zero thrust). In this scenario, FLOPS treats the three propulsor classes as one. This case is used as a verification case for the L0 mission analysis approach.

The same aerodynamic tables and the engine data set used in FLOPS were used in the L0 mission analysis approach. It is important to mention that FLOPS has internal interpolation and optimization routines, while the L0 approach relies on SciPy's algorithms. ${ }^{9}$ Also, the new approach does not require outside loops to compute the battery weight as required when using FLOPS.

As seen in Fig. 3, the two methods provide similar results. The small differences are possibly due to the different optimization and interpolation algorithms used. Figure 3(c) shows an offset in the overall vehicle weight. This is due to a small discrepancy in the battery weight computed in each case. The FLOPS approach computed a battery weight of $12335 \mathrm{lb}$; whereas the L0 approach resulted in a battery weight of $12416 \mathrm{lb}$. The discrepancy between approaches in this case is only $81 \mathrm{lb}$ ( $0.7 \%$ relative difference). These results provide confidence that, in its most basic form, the L0 mission analysis approach can replicate the behavior of FLOPS. The wall-clock time used by using FLOPS is about 5 to 10 seconds while the L0 approach takes about 20 to 30 seconds. This time difference is expected because FLOPS is a compiled code, while the L0 approach is written in an interpreted language (Python 3.x). The increase in wall-clock time is not significant when one considers the added analysis flexibility. 


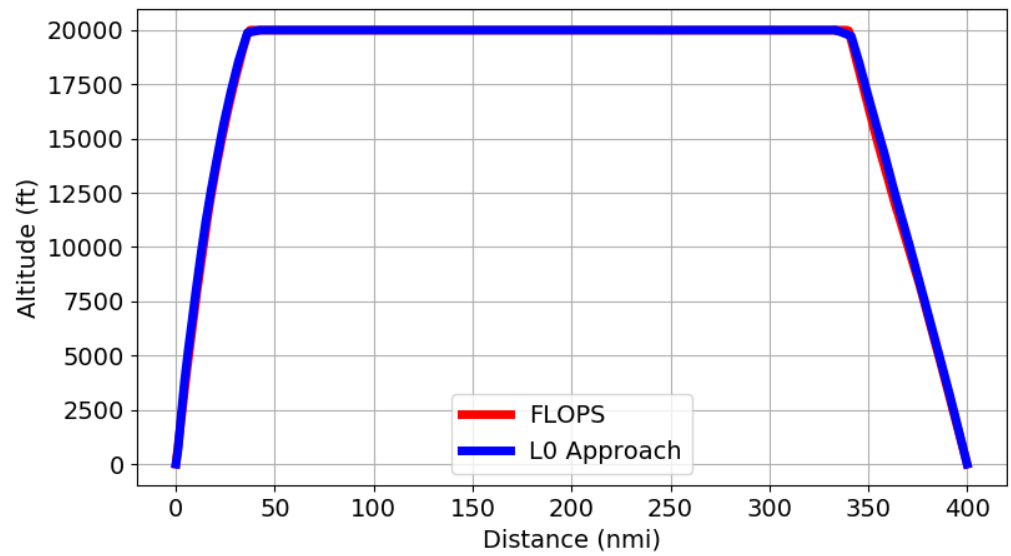

(a) Altitude versus distance traveled.

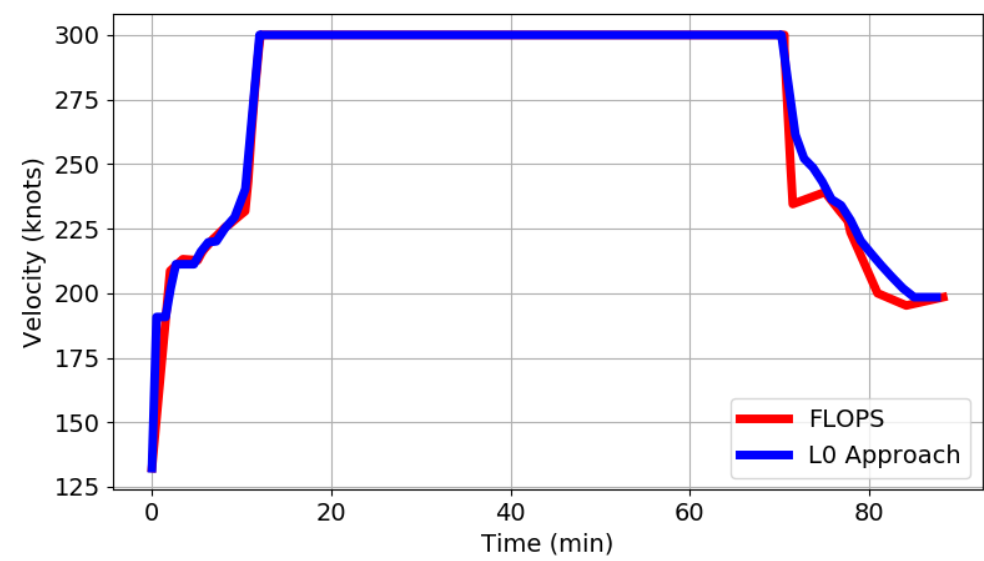

(b) Velocity versus time.

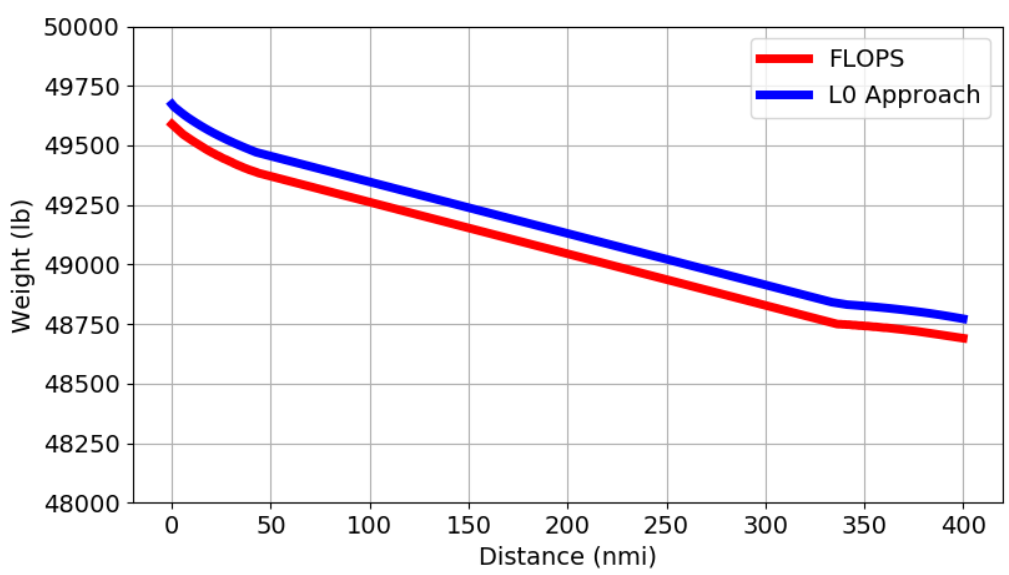

(c) Weight versus distance traveled.

Figure 3. PEGASUS trajectory using a single engine data set. 


\section{B. Multiple Propulsors}

As previously mentioned, FLOPS does not have the capability to throttle each propulsor independently. This is a major challenge because the split between fuel and electric energy has a large impact on the vehicle sizing. As discussed in Section III, the L0 mission analysis approach can throttle each propulsor independently. Two test cases in which each propulsor class has its own engine data set and can be throttled independently to minimize the desired objective function were studied. Each segment in the mission has different objective functions. The climb segment attempts to minimize the time to climb, the descent segment is a low powered descent that maximizes $L / D$. The objective function for the cruise segment of the first case (referred to as Case 1) is to minimize the electric energy used. The objective function for the cruise segment of the second case (referred to as Case 2) is to minimize the overall energy (electric and fuel) used.

The results in terms of energy usage are shown in Table 2. The single engine data set case discussed in the previous section is referred to as Case 0 and shown for comparison purposes. As expected, Case 1 has the lowest amount of electric energy used. This translates to a battery weight of 11,029 lb. In Case 2, the total energy used is decreased by decreasing the fuel used while increasing the electric energy with respect to Case 1. This resulted in a battery weight of $18,820 \mathrm{lb}$, a 7,791 lb difference with respect to Case 1 . The wall-clock time for these cases was about 40 to 60 seconds.

Table 2. Energy Usage for the Different Test Cases

\begin{tabular}{lccc}
\hline \hline & Case 0 & Case 1 & Case 2 \\
\hline Fuel Energy (kW-hr) & 4855 & 5195 & 3662 \\
Electric Energy (kW-hr) & 2253 & 2001 & 3414 \\
Total Energy (kW-hr) & 7108 & 7196 & 7077 \\
Fuel Used (lb) & 899 & 963 & 679 \\
Battery Weight (lb) & 12416 & 11029 & 18820 \\
\hline
\end{tabular}

The optimal trajectories flown in both test cases are shown in Fig. 4. The effects of the heavier battery weight in Case 2 are seen in the climb segment, where the vehicle takes longer to reach cruise conditions.

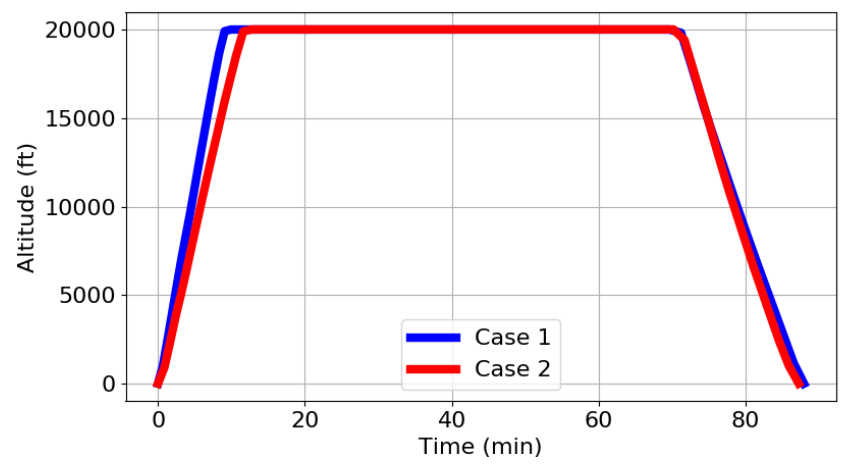

(a) Altitude versus time.

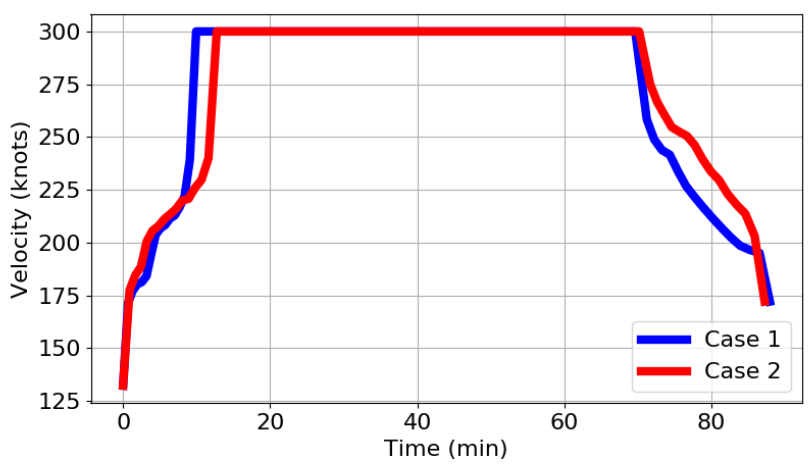

(b) Velocity versus time.

Figure 4. Trajectories for minimum electric and total minimum energy test cases. Case 1 minimizes the electric energy used. Case 2 minimizes the total energy used.

The trajectories are not identical, but are relatively similar. The main difference is in how the propulsors are being used throughout the mission. Figure 5 shows the throttle profile for both test cases. Case 1 clearly indicates that during cruise both electric propulsors must be off to minimize the electric energy. Case 2, on the other hand, uses both electric propulsors near their maximum throttle while decreasing the hybrid propulsor throttle. 


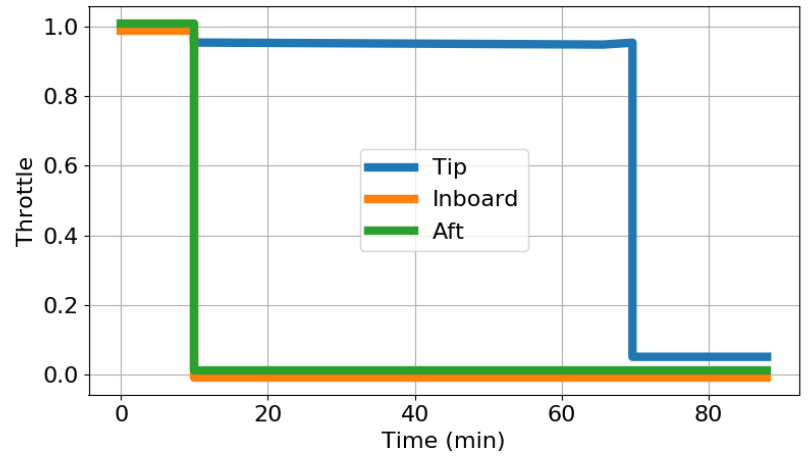

(a) Throttle profile for Case 1 .

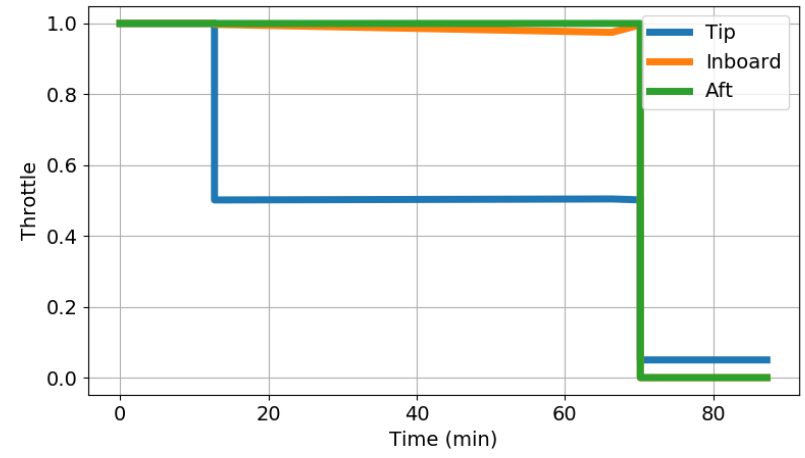

(b) Throttle profile for Case 2 .

Figure 5. Throttle profiles for Case 1 and Case 2. Case 1 minimizes the electric energy used. Case 2 minimizes the total energy used

It is important to mention that aerodynamic and propulsion interactions were not modeled in these cases, and the engine data sets are rudimentary. The results shown here are not used to make any conclusions regarding the PEGASUS concept but to show the capabilities of the L0 mission analysis approach.

\section{Conclusions}

This paper explored a low-order mission analysis approach (referred to as L0) currently being designed for the Layered and Extensible Aircraft Performance System (LEAPS). This approach is based on the method already used in the Flight Optimization Software (FLOPS) but has been extended to handle novel propulsion systems and a broader range of aircraft configurations. This novel mission analysis approach tends to require slightly more computational time than its FLOPS counterpart; however, the added flexibility suggests that this approach is appropriate for LEAPS.

The Parallel Electric-Gas Architecture with Synergistic Utilization Scheme (PEGASUS) concept was used to test the new low-order mission analysis approach. The first test case compared the L0 mission analysis approach with FLOPS when sizing the battery required to complete the mission. The results suggest that the L0 mission analysis approach and FLOPS provide similar outcomes when compared directly. This provides confidence that, if required, the L0 mission analysis approach can produce similar results to FLOPS.

The main goal of the L0 mission analysis approach is to expand the capabilities available in FLOPS. For this reason, two more test cases were used to explore the effects of throttling each propulsor class independently. The results suggest that, depending on the objective function used, the resulting battery weight and fuel used can vary substantially. This new capability provides the analyst with extra degrees of freedom when sizing novel concepts.

Overall, the L0 mission analysis approach has the capability to replicate FLOPS, when needed, but can also size electric and hybrid-electric aircraft without the need for complex workarounds. This approach, coupled with the flexibility of LEAPS, will enable the analyst to perform the analysis of novel vehicles in a timely manner (with respect to FLOPS) while providing more design degrees of freedom. These could ultimately translate to better designs without the need to have large computational resources.

\section{Acknowledgments}

We would like to thank Mark Guynn (NASA Langley Research Center) for his technical support and Kevin Antcliff (NASA Langley Research Center) for the many discussions regarding PEGASUS and its operational modes. This work was funded by the NASA Transformational Tools \& Technologies (TTT) project. 


\section{References}

${ }^{1}$ Welstead, J. R., Caldwell, D., Condotta, R., and Nerissa, M., "An Overview of the Layered and Extensible Aircraft Performance System (LEAPS) Development," To be Published in SciTech 2018, 2018.

${ }^{2} \mathrm{McCullers}, \mathrm{L}$. A., "Aircraft Configuration Optimization including Optimized Flight Profiles," NASA. Langley Research Center Recent Experiences in Multidisciplinary Analysis and Optimization, Part 1., Hampton, VA, 1984.

${ }^{3}$ McMullers, L., Flight Optimization System, FLOPS. User's Guide, release 8.23, Hampton, VA, 2011.

${ }^{4}$ Capristan, F. M. and Welstead, J., "LEAPS: An Initial Assessment Towards a Multi-Order Approach to Air Vehicle Mission Analysis," 18th AIAA/ISSMO Multidisciplinary Analysis and Optimization Conference, AIAA AVIATION Forum, American Institute of Aeronautics and Astronautics, Jun 2017, doi:10.2514/6.2017-4325.

${ }^{5}$ Rutowski, E. S. and Company, D. A., "Energy Approach to the General Aircraft Performance Problem," Journal of The Aeronautical Sciences, 1954.

${ }^{6}$ Daniel Raymer, Aircraft Design: A Conceptual Approach, American Institute of Aeronautics and Astronautics, 5th ed., 2012.

${ }^{7}$ Antcliff, K. R., Guynn, M. D., Marien, T., Wells, D. P., Schneider, S. J., and Tong, M. J., "Mission Analysis and Aircraft Sizing of a Hybrid-Electric Regional Aircraft," 54th AIAA Aerospace Sciences Meeting, AIAA SciTech, American Institute of Aeronautics and Astronautics, Jan 2016, doi:10.2514/6.2016-1028.

${ }^{8}$ Antcliff, K. R. and Capristan, F. M., "Conceptual Design of the Parallel Electric-Gas Architecture with Synergistic Utilization Scheme (PEGASUS) Concept," 18th AIAA/ISSMO Multidisciplinary Analysis and Optimization Conference, AIAA AVIATION Forum, American Institute of Aeronautics and Astronautics, Jun 2017, doi:10.2514/6.2017-4001.

${ }^{9}$ Jones, E., Travis, O., and Peterson, P., "SciPy: Open source scientific tools for Python," http://www.scipy.org, 2001, [Online; accessed 04-21-2017].

${ }^{10} \mathrm{Kraft}$, D., "A software package for sequential quadratic programming," Forschungsbericht- Deutsche Forschungs- und Versuchsanstalt fur Luft- und Raumfahrt, 1988. 